\title{
Small Intestinal Bacterial Overgrowth: Diagnosis and Treatment
}

\author{
Antonio Gasbarrini Ernesto Cristiano Lauritano Maurizio Gabrielli \\ Emidio Scarpellini Andrea Lupascu Veronica Ojetti Giovanni Gasbarrini \\ Internal Medicine Department, Gemelli Hospital, Catholic University of Sacred Heart, Rome, Italy
}

\section{Key Words}

Small intestinal bacterial overgrowth $\cdot$ Antibiotics $\cdot$ Breath tests $\cdot$ Irritable bowel syndrome

\begin{abstract}
Small intestinal bacterial overgrowth (SIBO) is a clinical condition characterized by a malabsorption syndrome due to an increase in microorganisms within the small intestine. The main mechanisms restricting bacterial colonization in the upper gut are the gastric acid barrier, mucosal and systemic immunity and intestinal clearance. When these mechanisms fail, bacterial overgrowth develops. Diarrhea, steatorrhea, chronic abdominal pain, bloating and flatulence are common symptoms and are similar to those observed in irritable bowel syndrome. Breath tests (glucose and/or lactulose breath tests) have been proposed as a sensitive and simple tool for the diagnosis of bacterial overgrowth, being noninvasive and inexpensive compared to the gold standard represented by the culture of intestinal aspirates. Antibiotic therapy is the cornerstone of SIBO treatment. Current SIBO treatment is based on empirical courses of broad-spectrum antibiotics since few controlled studies concerning the choice and duration of antibiotic therapy are available at present.

Copyright $\odot 2007$ S. Karger AG, Basel
\end{abstract}

(C) 2007 S. Karger AG, Basel

0257-2753/07/0253-0237\$23.50/0

Fax +4161306 1234

E-Mail karger@karger.ch

www.karger.com
Accessible online at: www.karger.com/ddi

\section{Introduction}

Adult humans live in symbiosis with several bacteria species exceeding the number of host somatic cells by at least one order of magnitude [1]. Intestinal microflora is a complex microbiological system composed of parasites, viruses, yeast and, above all, bacteria. The bacterial concentration varies along the gastrointestinal tract increasing from $10^{3}$ colony-forming units (CFU)/ $\mathrm{ml}$ in the upper intestinal tract to $10^{14} \mathrm{CFU} / \mathrm{ml}$ in the colon.

Intestinal ecoflora is responsible for integrity and function of the gastrointestinal tract. It plays a role in the defense from pathogenic microorganisms, in the stimulation of the immune system, in the control of metabolic and trophic function of epithelial cells and in the synthesis of vitamins and nutrients [2]. It also exerts remarkable effects in the development and maintenance of gut sensory and motor functions, including the promotion of intestinal propulsive activity.

There is emerging evidence indicating that quantitative and qualitative changes in intestinal flora contribute to the pathogenesis of intestinal and extraintestinal diseases. Small intestinal bacterial overgrowth (SIBO) is a condition associated with the presence of $>10^{6} \mathrm{CFU} / \mathrm{ml}$ of intestinal aspirate and/or the presence of colonic-type species [3]. Normally, SIBO is prevented by action of the

Prof. Antonio Gasbarrin

Internal Medicine Department, Catholic University of Sacred Heart Gemelli Hospital, Largo A. Gemelli, 8

IT-00168 Rome (Italy)

Tel. +39 063015 4294, Fax +39063550 2775, E-Mail angiologia@rm.unicatt.it 
intestinal immune system, gastric acid, pancreatic enzymes, small intestinal motility and the ileocecal valve. When one or more of these mechanisms fail, SIBO can occur.

\section{Metabolic Effects of Bacterial Overgrowth}

Bacteria in excess can interfere with the metabolism and the absorption of many substances such as carbohydrates, proteins, lipids and vitamins. The loss of activity of brush-border disaccharidases due to mucosal injury and the bacteria fermentation of sugars such as sorbitol, fructose and lactose could be responsible for carbohydrate malabsorption [4]. Enterocyte injury may alter the gut permeability, predisposing to the development of a protein-losing enteropathy. Moreover, bacteria may compete with the host for protein and lead to the production of ammonia [5]. Deconjugation of bile acids in the proximal gut induces fat and lipophilic vitamin (A, D, E) malabsorption and leads to the production of lithocholic acid, which is poorly absorbed and may be directly toxic to enterocytes [6]. Cobalamin (vitamin $\mathrm{B}_{12}$ ) deficiency can occur in SIBO as a result of use of the vitamin by anaerobic bacteria. Levels of both folates and vitamin $\mathrm{K}$, however, are usually normal or increased in SIBO as a result of bacterial production.

\section{Clinical Aspects of Bacterial Overgrowth}

Small intestinal bacterial overgrowth is generally considered a malabsorption syndrome, although clinical manifestations can be largely different in each subject. This variability is caused by many factors including the entity of contamination, the extension of intestinal tract, the predisposing factors causing SIBO and the bacterial species involved. Common SIBO symptoms are diarrhea, steatorrhea, chronic abdominal pain, bloating and flatulence, although asymptomatic cases have been described [7].

SIBO symptoms are similar to those observed in patients affected by irritable bowel syndrome (IBS). Recent findings suggest that SIBO could play a role in the pathogenesis and clinical manifestations of IBS and eradication of SIBO is associated with a significant improvement of IBS symptoms [8, 9]. However, further studies are needed to confirm this clinical association. Unusual SIBO presentations include megaloblastic anemia, osteomalacia, neuropathy, weight loss and peripheric edema.

\section{Diagnosis of Bacterial Overgrowth}

Aspiration and direct culture of jejunal contents are considered the gold standards for the diagnosis of SIBO [10]. These procedures have some limitations, such as invasivity, possible contamination by oropharyngeal bacteria, low reproducibility and presence of non-culturable bacteria. For this reason, non-invasive tests are commonly used for the diagnosis of SIBO (breath tests). These are based on production of hydrogen and methane by bacteria as a consequence of carbohydrate fermentation [11]. The diagnosis of SIBO is established when the exhaled hydrogen level increases by $>10$ parts per million greater than baseline (for glucose breath test) or when though double peaks (SIBO and colonic peaks) have been clearly found after lactulose ingestion. The specificity and sensitivity of breath tests are not excellent but they are a noninvasive, simple and inexpensive tool for the diagnosis of bacterial overgrowth.

\section{Treatment of Bacterial Overgrowth}

SIBO therapy is based on two different approaches: treatment of predisposing conditions and antibiotic administration. Little evidence exists for the efficacy of prokinetics and probiotics in SIBO treatment.

\section{Antibiotics}

Although ideally the antibiotic choice should reflect in vitro susceptibility testing, this is usually difficult in SIBO because of the presence of several bacterial species with different antibiotic sensitivities. Therefore, antibiotic treatment requires the administration of wide-spectrum antibiotics, also if the best pharmacological approach in terms of drug, dosage and duration of therapy remains to be assessed.

Tetracyclines have been used for a long time; however, this class of drugs is associated with several side effects and a low eradication rate (about 30\%) since they do not have a direct activity against anaerobes and may be ineffective against bacteroides [12].

Metronidazole has been used with satisfying results as an alternative to tetracycline. In a study on patients with blind-loop syndrome, metronidazole showed a higher therapeutic efficacy than a non-absorbable antibiotic, rifaximin [13]. 
Attar et al. [14] compare the efficacy of amoxicillinclavulanic acid, norfloxacin, and Saccharomices boulardii for the treatment of SIBO-related diarrhea. A statistically significant improvement in mean daily number of stools was obtained with norfloxacin (90\%) and amoxicillin-clavulanic acid (60\%), and none with S. boulardii. A study by Castiglione et al. [15] found a good therapeutic efficacy of both metronidazole and ciprofloxacin in terms of SIBO eradication in patients affected by Crohn's disease. Ciprofloxacin showed a small, but not statistically significant gain in terms of efficacy and tolerability compared to metronidazole. Some authors evaluated the therapeutic efficacy of non-absorbable antibiotics such as rifaximin and neomycin in order to minimize the potential side effects of systemic antibiotics.

Data on rifaximin, a rifamycin derivative with antibacterial activity caused by inhibition of bacterial synthesis of RNA, show a bactericidal action against both aerobes and anaerobes, such as bacterioides, lactobacilli and clostridia [16]. Less than $0.1 \%$ of the oral dose of rifaximin is absorbed, therefore it exhibits less toxicity than other antibiotics. In a double-blind controlled trial, Di Stefano et al. [12] compared the efficacy of rifaximin $(1,200 \mathrm{mg} /$ day $)$ with respect to chlortetracycline in the short-term treatment of SIBO. The glucose breath test normalized in $70 \%$ of patients treated with rifaximin versus $27 \%$ of patients treated with chlortetracycline. No patient showed any side effect in the rifaximin group. Recently, Lauritano et al. [17] showed that higher doses of rifaximin $(1,200 \mathrm{mg} /$ day $)$ were associated with a significantly higher therapeutic efficacy $(60 \%$ of glucose breath test normalization) in terms of SIBO eradication with respect to doses of $600 \mathrm{mg} /$ day $(16.7 \%$ of glucose breath test normalization) and $800 \mathrm{mg} /$ day (26.7\% of glucose breath test normalization). Similarly, Cuoco et al. [18] assessed the efficacy of rifaximin (1,200 $\mathrm{mg} /$ day), followed by a 20 -day course of probiotics, in the treatment of SIBO. The eradication rate of this schedule achieved $83 \%$ with a significant improvement of gastrointestinal symptoms.

Neomycin, a non-absorbable aminoglycoside, was shown to be of little efficacy when used alone in SIBO. In a recent study by Pimentel et al. [19] on 111 IBS patients, treatment with neomycin achieved the normalization of lactulose breath test in $20 \%$ of patients with SIBO with respect to $2 \%$ in the placebo group. No relevant side effects were observed during the study and no dropouts occurred. Given these data, there is no conclusive information regarding the most effective therapy that should be used in the treatment of SIBO. Treatment decisions should be individualized and consider risks of long-term antibiotic therapy (diarrhea, Clostridium difficile infection, intolerance, bacterial resistance, costs) and the possibility of SIBO recurrence.

\section{References}

1 Luckey TD: Introduction to intestinal microecology. Am J Clin Nutr 1972;25:12921294.

2 Hooper LV, Midtvedt T, Gordon JI: How host-microbial interactions shape the nutrient environment of the mammalian intestine. Annu Rev Nutr 2002;22:283-307.

-3 Donaldson RM Jr: Normal bacterial population of the intestine and their relation to intestinal function. N Engl J Med 1964;270: 938-945.

-4 Nucera G, Gabrielli M, Lupascu A, Lauritano EC, Santoliquido A, Cremonini F, Cammarota G, Tondi P, Pola P, Gasbarrini G, Gasbarrini A: Abnormal breath tests to lactose, fructose and sorbitol in irritable bowel syndrome may be explained by small intestinal bacterial overgrowth. Aliment Pharmacol Ther 2005;21:1391-1395.

5 Varcoe R, Haliday D, Tavill AS: Utilization of urea nitrogen for albumin synthesis in the stagnant loop syndrome: Gut 1974;15:898902.
6 Wanitschke R, Ammon HV: Effects of dihydroxy bile acids and hydroxy fatty acids on the absorption of oleic acid in the human jejunum. J Clin Invest 1978;61:178-186.

$\checkmark 7$ Saltzman JR, Kowdley KV, Pedrosa MC, Sepe T, Golner B, Perrone G, Russell RM: Bacterial overgrowth without clinical malabsorption in elderly hypochlorhydric subjects. Gastroenterology 1994;106:615-623.

${ }_{8}$ Lin EC: Small intestinal bacterial overgrowth, a framework for understanding irritable bowel syndrome. JAMA 2004;292: 852-858.

-9 Lupascu A, Gabrielli M, Lauritano EC, Scarpellini E, Santoliquido A, Cammarota G, Flore R, Tondi P, Pola P, Gasbarrini G, Gasbarrini A: Hydrogen glucose breath test to detect small intestinal bacterial overgrowth: prevalence case-control study in irritable bowel syndrome. Aliment Pharmacol Ther 2005;22:1157-1160.
10 Corazza GR, Menozzi MG, Strocchi A, Rasciti L, Vaira D, Lecchini R, Avanzini P, Chezzi C, Gasbarrini G: The diagnosis of small bowel bacterial overgrowth. Gastroenterology 1990;98:302-309.

11 Romagnuolo J, Schiller D, Bailey RJ: Using breath tests wisely in a gastroenterology practice: an evidence-based review of indications and pitfalls in interpretation. Am J Gastroenterol 2002;97:1113-1126.

12 Di Stefano M, Malservisi S, Veneto G, Ferrieri A, Corazza GR: Rifaximin versus chlortetracycline in the short-term treatment of small intestinal bacterial overgrowth. Aliment Pharmacol Ther 2000;14:551-556.

13 Di Stefano M, Miceli E, Missanelli A, Mazzochi S, Corazza GR: Absorbable vs. non-absorbable antibiotics in the treatment of small intestine bacterial overgrowth in patients with blind-loop syndrome. Aliment Pharmacol Ther 2005;21:985-992. 
14 Attar A, Flourie B, Rambaud JC, Franchisseur C, Ruszniewski P, Bouhnik Y: Antibiotic efficacy in small intestinal bacterial overgrowth related chronic diarrhea: a crossover, randomized trial. Gastroenterology 1999;117:794-797.

15 Castiglione F, Rispo A, Di Girolamo E, Cozzolino A, Manguso F, Grassia R, Mazzacca G: Antibiotic treatment of small bowel bacterial overgrowth in patients with Crohn's disease. Aliment Pharmacol Ther 2003;18: 1107-1112.
16 Scarpignato C, Pelosini I: Experimental and clinical pharmacology of rifaximin, a gastrointestinal selective antibiotic. Digestion 2006;73(suppl 1):13-27.

17 Lauritano EC, Gabrielli M, Lupascu A, Santoliquido A, Nucera G, Scarpellini E, Vincenti F, Cammarota G, Flore R, Pola P, Gasbarrini G, Gasbarrini A: Rifaximin dose-finding study for the treatment of small intestinal bacterial overgrowth. Aliment Pharmacol Ther 2005;22:31-35.
8 Cuoco L, Salvagnini M: Small intestine bacterial overgrowth in irritable bowel syndrome: a retrospective study with rifaximin. Minerva Gastroenterol Dietol 2006;52:8995.

19 Pimentel M, Chow EJ, Lin HC: Normalization of lactulose breath testing correlates with symptom improvement in irritable bowel syndrome: a double-blind, randomized, placebo-controlled study. Am J Gastroenterol 2003;98:412-419. 